\title{
First detection of Hydrogen Chloride towards protostellar shocks
}

\author{
C. Codella ${ }^{1}$, C. Ceccarelli ${ }^{2}$, S. Bottinelli ${ }^{3}$, M. Salez ${ }^{4}$, S. Viti ${ }^{5}$, B. Lefloch ${ }^{2,6}$, S. Cabrit ${ }^{4}$, E.

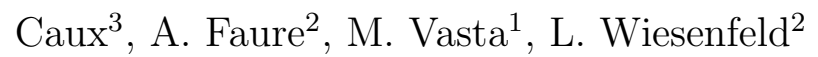

Received - ; accepted -

\begin{abstract}
We present the first detection of hydrogen chlorine in a protostellar shock, by observing the fundamental transition at $626 \mathrm{GHz}$ with the Herschel HIFI spectrometer. We detected two of the three hyperfine lines, from which we derived a line opacity $\leq 1$. Using a non-LTE LVG code, we constrained the $\mathrm{HCl}$ column density, temperature and density of the emitting gas. The hypothesis that the emission originates in the molecular cloud is ruled out, as it would imply a too dense gas. Conversely, assuming that the emission originates in the $10^{\prime \prime}-15^{\prime \prime}$ size shocked gas previously observed at the IRAM PdB interferometer, we obtain: $\mathrm{N}(\mathrm{HCl})=0.7-2 \times 10^{13} \mathrm{~cm}^{-2}$, temperature $>15 \mathrm{~K}$ and density $>3 \times 10^{5} \mathrm{~cm}^{-3}$. Combining with the Herschel HIFI $\mathrm{CO}(5-4)$ observations allows to further constrain the gas density and temperature, $10^{5}-10^{6} \mathrm{~cm}^{-3}$ and $120-250 \mathrm{~K}$, as well as the $\mathrm{HCl}$ column density, $2 \times 10^{13} \mathrm{~cm}^{-2}$, and, finally, abundance: $\sim 3-6 \times 10^{-9}$. The estimated $\mathrm{HCl}$ abundance is consistent with that previously observed in lowand high- mass protostars. This puzzling result in the L1157-B1 shock, where species from volatile and refractory grains components are enhanced, suggests either that $\mathrm{HCl}$ is not the main reservoir of chlorine in the gas phase, against previous chemical models predictions, or that the elemental chlorine abundance
\end{abstract}

\footnotetext{
${ }^{1}$ INAF, Osservatorio Astrofisico di Arcetri, Largo Enrico Fermi 5, I-50125 Firenze, Italy codella@rcetri.astro.it

${ }^{2}$ UJF-Grenoble 1 / CNRS-INSU, Institut de Planétologie et d'Astrophysique de Grenoble (IPAG) UMR 5274, Grenoble, F-38041, France

${ }^{3}$ Université de Toulouse; UPS-OMP; Toulouse, France; CNRS, IRAP, 9 Av colonel Roche, BP44346, F-31028, Toulouse, France

${ }^{4}$ Observatoire de Paris-Meudon, LERMA UMR CNRS 8112. Meudon, France

${ }^{5}$ Department of Physics and Astronomy, University College London, London, UK

${ }^{6}$ Centro de Astrobiologia, CSIC-INTA, Carretera de Ajalvir, Km 4, Torrejon de Ardoz, 28850, Madrid, Spain
} 
is low in L1157-B1. Astrochemical modelling suggests that $\mathrm{HCl}$ is in fact formed in the gas phase, at low temperatures, prior to the occurance of the shock, and that the latter does not enhance its abundance.

Subject headings: ISM: jets and outflows — ISM: molecules — ISM: abundances

\section{Introduction}

During the earliest protostellar stages of their evolution, Sun-like stars generate fast jets and wide angle winds impacting against the high-density parent cloud and creating shock fronts. The ambient gas is heated and compressed and several chemical formation routes open up. Several molecular species, such as $\mathrm{H}_{2} \mathrm{O}$, undergo spectacular enhancements by orders of magnitude in their abundances (Liseau et al. 1996), as actually also observed at mm and submm wavelengths (e.g. Garay et al. 1998; Bachiller \& Pérez Gutiérrez 1997). Given that protostellar winds transfer momentum and energy back to the ambient medium, studies of the chemical composition of shocked regions are essential in understanding not only the chemistry but also the energetics of the process.

The L1157 region, located at 250 pc, hosts a Class 0 protostar (L1157-mm) driving a spectacular chemically rich bipolar outflow (Bachiller et al. 2001, and references therein), which is considered as one of the best laboratories where to study how shocks affect the molecular gas. The L1157-mm outflow is associated with several bow shocks seen in the IR $\mathrm{H}_{2}$ lines (e.g. Neufeld et al. 2009) and in the CO lines (Gueth et al. 1996). These bow shocks, mapped with the IRAM PdB interferometer, reveal a clumpy structure, with the clumps located at the wall of the cavity opened by the jet (e.g. Benedettini et al. 2007; Codella et al. 2009). As part of the Hersche 1 Key Program CHESS2 (Chemical Herschel Surveys of Star forming regions; Ceccarelli et al. 2010), the brightest bow-shock called L1157-B1, is currently being investigated with a spectral survey in the $\sim 500-2000 \mathrm{GHz}$ interval using the Herschel HIFI instrument (de Graauw et al. 2010). Preliminary results (Codella et al. 2010, Lefloch et al. 2010) have confirmed the chemical richness of L1157$\mathrm{B} 1$ and revealed the presence of different molecular components with different excitation conditions coexisting in the B1 bow structure.

The Cl chemistry has been investigated by several authors (Schilke et al. 1995; Neufeld

\footnotetext{
${ }^{1}$ Herschel is an ESA space observatory with science instruments provided by European-led principal Investigator consortia and with important partecipation from NASA.

${ }^{2}$ http://www-laog.obs.ujf-grenoble.fr/heberges/chess/
} 
\& Wolfire 2009, and references therein), indicating hydrogen chloride ( $\mathrm{HCl}$ ) as the most abundant Cl-bearing molecule in dense interstellar clouds. The CHESS survey of L1157-B1 offers an excellent opportunity to observe emission from $\mathrm{HCl}$ towards shocked gas. The $\mathrm{HCl} J=1-0$ transition falls at $626 \mathrm{GHz}$, where a strong atmospheric water absorption is present, and it is therefore observable from ground only under exceptional weather conditions and towards bright sources. As a matter of fact, observations from ground have so far been obtained towards OMC-1 (Blake et al. 1985; Schilke et al. 1995; Salez et al. 1996; Neufeld \& Green 1994), and, very recently, towards a sample of high- and low- mass star forming regions (Peng et al. 2010). On the contrary, Herschel has already provided clear detections of $\mathrm{HCl}$ towards the massive star formation W3A and the carbon-rich star IRC+10216 (Cernicharo et al. 2010a, 2010b) in its first two months of life.

\section{Observations and data reduction}

The $\mathrm{H}^{37} \mathrm{Cl}$ and $\mathrm{H}^{35} \mathrm{Cl} 1-0$ transitions at 624.978 and $625.902 \mathrm{GHz}$ were observed towards L1157-B1 on 2010, June 23rd (pointed observation OBS_1342199173) with HIFI at the position $\alpha_{\mathrm{J} 2000}=20^{\mathrm{h}} 39^{\mathrm{m}} 10^{\mathrm{s}} .2 \delta_{\mathrm{J} 2000}=+68^{\circ} 01^{\prime} 10^{\prime \prime} .5$. We also observed the ${ }^{13} \mathrm{CO}(5-$ 4) spectrum at $551 \mathrm{GHz}$ on 2010, 27th October (OBS_1342207575), during the unbiased spectral survey of the band called 1a of HIFI. The observations were carried out in double beam switching mode. The receiver was tuned in double side band, with a total integration time of 34.6 min for $\mathrm{HCl}$ and 148.5 min to cover band 1a, respectively. For $\mathrm{HCl}$, both the Wide Band Spectrometer (WBS) and the High Resolution Spectrometer (HRS) were used in parallel, while for ${ }^{13} \mathrm{CO}(5-4)$ pnly the WBS has been used. The velocity resolution is 0.24 and $0.27 \mathrm{~km} \mathrm{~s}^{-1}$ for $\mathrm{HCl}$ and ${ }^{13} \mathrm{CO}(5-4)$, respectively. Both $\mathrm{HCl}$ transitions are split into three hyperfine components, whose frequencies are given in Table 1. Fluxes are expressed in units of main-beam temperature $T_{\mathrm{mb}}$. The HPBW is $34^{\prime \prime}$ and $39^{\prime \prime}$ at the frequencies of the $\mathrm{HCl}(1-0)$ and ${ }^{13} \mathrm{CO}(5-4)$ lines, respectively. The main-beam efficiency $\left(\eta_{m b}\right)$ is 0.75 , as determined from beam observations towards Mars.

The data were processed with the ESA-supported package HIPE 3 (Herschel Interactive Processing Environment). Fits files from level 2 were then created and transformed into GILDAS 4 format for baseline subtraction and subsequent data analysis. Both polarisations $\mathrm{H}$ and $\mathrm{V}$ were reduced and analysed separately.

\footnotetext{
${ }^{3} \mathrm{HIPE}$ is a joint development by the Herschel Science Ground Segment Consortium, consisting of ESA, the NASA Herschel Science Center, and the HIFI, PACS and SPIRE consortia.

${ }^{4} \mathrm{http}: / /$ www.iram.fr/IRAMFR/GILDAS
} 

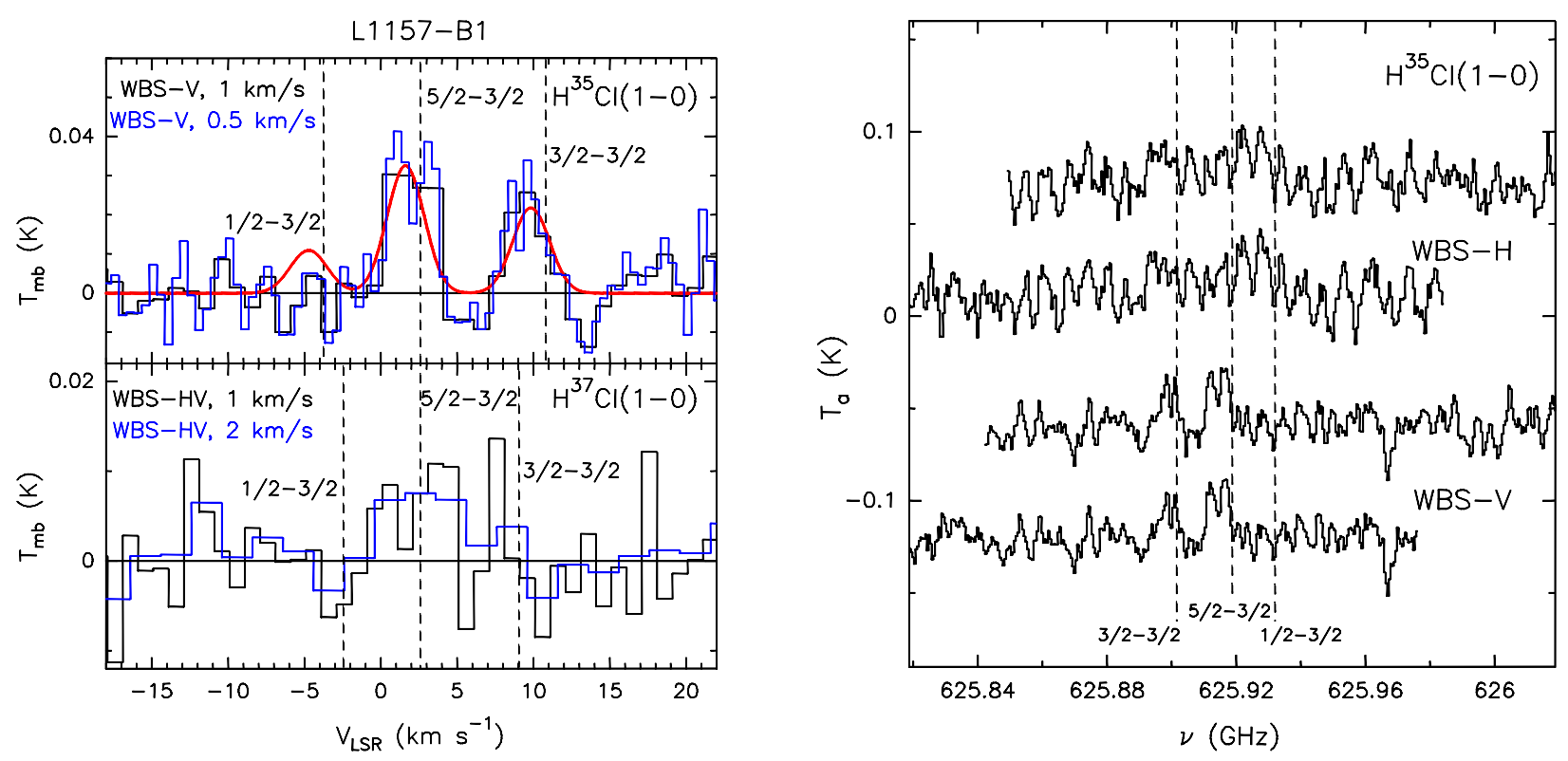

Fig. 1.- $\mathrm{H}^{35} \mathrm{Cl}(1-0)$ and $\mathrm{H}^{37} \mathrm{Cl}(1-0)$ spectra (in $T_{\mathrm{MB}}$ scale) observed towards the L1157-B1 clump. The observed coordinates are $\alpha_{\mathrm{J} 2000}=20^{\mathrm{h}} 39^{\mathrm{m}} 10^{\mathrm{s}} \cdot 2, \delta_{\mathrm{J} 2000}=+68^{\circ} 01^{\prime} 10^{\prime \prime} .5$, i.e. at $\Delta \alpha=+25$ ". 6 and $\Delta \delta=-63^{\prime \prime} .5$ from the driving protostar. Each transition is split into three hyperfine components whose frequencies (Table 1) are pointed out by dashed vertical lines. The red curve is for the fit performed using the GILDAS CLASS tool (Table 1). Upper-Left panel: $\mathrm{H}^{35} \mathrm{Cl}(1-0)$ profiles observed with the HIFI WBS spectrometer in V-polarisation (Sect. 2) and at a spectral resolution of 0.5 and $1 \mathrm{~km} \mathrm{~s}^{-1}$. Lower-Left panel: $\mathrm{H}^{37} \mathrm{Cl}(1-0)$ profiles observed with the HIFI WBS spectrometer and a spectral resolution of 1 and $2 \mathrm{~km} \mathrm{~s}^{-1}$. In this case, the $\mathrm{H}$ - and V-polarisations have been averaged to gain sensitivity (Sect. 2). Right panel: Comparison of the WBS subscans (in $T_{\mathrm{a}}$ scale) at the $\mathrm{H}^{35} \mathrm{Cl}(1-0)$ frequencies: two subscans in $\mathrm{V}$-polarisation where the $\mathrm{H}^{35} \mathrm{Cl}(1-0)$ profile is already detectable (lower spectra), and two subscans in H-polarisation (upper spectra) where the spectra is affected by ripples and consequently higher noise. 


\section{Results: $\mathrm{HCl}$ emission at low velocities}

The V-spectra observed with the WBS backend are shown in Fig. 1, where we report the profile with a spectral resolution of $0.5 \mathrm{~km} \mathrm{~s}^{-1}$, and the profile smoothed to $1 \mathrm{~km} \mathrm{~s}^{-1}$ to increase sensitivity. The V-spectra are characterised by a flat baseline with an $\mathrm{rms}$ of $6 \mathrm{mK}$ per frequency interval of $0.5 \mathrm{MHz}$. Two hyperfine components $(F=5 / 2-3 / 2$ and $F=3 / 2-3 / 2)$ of the $\mathrm{H}^{35} \mathrm{Cl}(1-0)$ line are detected in the sub-bands of the WBS, with intensities $\left(T_{\mathrm{mb}}\right)$ of $35 \mathrm{mK}$ and $27 \mathrm{mK}$ and linewidths of about $3 \mathrm{~km} \mathrm{~s}^{-1}$, i.e. they are detected at a level of $6 \sigma$ and $\sim 5 \sigma$, respectively. The weakest hyperfine component $(F=1 / 2-3 / 2)$ is not detected with a $3 \sigma$ sensitivity of $18 \mathrm{mK}$.

On the other hand, the H-spectrum is affected by strong ripples at the border of the subbands of the spectrometer, where the $\mathrm{H}^{35} \mathrm{Cl}$ line is located. These ripples, reported in Fig. 1, strongly degrade the quality of the baseline and result in an rms twice as large $(13 \mathrm{mK})$ as the one measured in the $\mathrm{V}$-spectrum. Therefore, in the following, we will analyse the $\mathrm{H}^{35} \mathrm{Cl}(1-0)$ emission based solely on the V-spectrum. All the parameters of the hyperfine components,

summarised in Table 1, were measured from a Gaussian fit of the whole $\mathrm{H}^{35} \mathrm{Cl}(1-0)$ pattern assuming a common linewidth and LSR velocity; the integrated intensity $\left(F_{\text {int }}\right)$ has been derived by summing the intensity of all the channel bins in the whole emitting velocity range for each hyperfine component.

The present data-set covers also the frequencies of the $\mathrm{H}^{37} \mathrm{Cl}(1-0)$ triplets (Table 1). For these frequencies we can use both $\mathrm{H}$ - and $\mathrm{V}$-polarisation spectra increasing the final sensitivity. The $\mathrm{H}^{37} \mathrm{Cl}(1-0)$ spectrum at a velocity resolution of $1 \mathrm{~km} \mathrm{~s}^{-1}$ (Fig. 1) puts a $3 \sigma$ upper limit of $15 \mathrm{mK}$ on the intensity of the hyperfine components. The $\mathrm{H}^{37} \mathrm{Cl}$ non-detection is in agreement with the intensities of the $\mathrm{H}^{35} \mathrm{Cl}(1-0)$ spectrum and optically thin emission given the solar ${ }^{35} \mathrm{Cl} /{ }^{37} \mathrm{Cl}$ abundance ratio of $\sim 3.1$ (Anders \& Grevesse 1989). Interestingly, once the spectral resolution is degraded to $2 \mathrm{~km} \mathrm{~s}^{-1}$ (Fig. 1), an emission bump of $\simeq 8 \mathrm{mK}$ is observed at the frequency of the $F=5 / 2-3 / 2$ hyperfine component. Unfortunately, the sensitivity $(1 \sigma=3 \mathrm{mK})$ is not enough to assess a firm detection.

Figure 2 compares the $\mathrm{H}^{35} \mathrm{Cl}(1-0) \quad F=5 / 2-3 / 2$ profile with the $\mathrm{H}_{2} \mathrm{O}\left(1_{10}-1_{01}\right)$ one observed by Lefloch et al. (2010) and Codella et al. (2010). Although the $\mathrm{S} / \mathrm{N}$ ratio of the $\mathrm{H}^{35} \mathrm{Cl}(1-0)$ lines does not allow us a proper study of the line profile, it clearly shows that $\mathrm{H}^{35} \mathrm{Cl}$ and $\mathrm{H}_{2} \mathrm{O}$ are tracing different gas: while water is associated with shocked gas at high velocities (up to $30 \mathrm{~km} \mathrm{~s}^{-1}$ with respect to the systemic velocity, $+2.6 \mathrm{~km} \mathrm{~s}^{-1}$ ), the $\mathrm{H}^{35} \mathrm{Cl}$ emission comes from the low velocity range $\left(\mathrm{FWHM} \simeq 3 \mathrm{~km} \mathrm{~s}^{-1}\right)$, suggesting that $\mathrm{H}^{35} \mathrm{Cl}$ is not a product of the shock. 
Table 1. List of molecular species and transitions here reported. Peak velocity, intensity (in $T_{\mathrm{mb}}$ scale), FWHM linewidth, integrated intensity $\left(F_{\text {int }}\right)$, as well as total column densities are listed. $\mathrm{For} \mathrm{H}^{35} \mathrm{Cl}(1-0)$ we report also the sum of the opacity of the hyperfine components $\left(\sum_{\mathrm{i}} \tau_{\mathrm{i}}\right)$, as provided by the GILDAS CLASS tool.

\begin{tabular}{|c|c|c|c|c|c|c|c|c|c|c|}
\hline Transition & $\begin{array}{c}\nu_{0}{ }^{a} \\
(\mathrm{MHz})\end{array}$ & $\begin{array}{l}E_{\mathrm{u}}{ }^{a} \\
(\mathrm{~K})\end{array}$ & $S$ & $\begin{array}{l}T_{\text {peak }} \\
(\mathrm{mK})\end{array}$ & $\begin{array}{l}\mathrm{rms}^{b} \\
(\mathrm{mK})\end{array}$ & $\begin{array}{c}V_{\text {peak }} \\
\left(\mathrm{km} \mathrm{s}^{-1}\right)\end{array}$ & $\begin{array}{l}F W H M \\
\left(\mathrm{~km} \mathrm{~s}^{-1}\right)\end{array}$ & $\sum_{\mathrm{i}} \tau_{\mathrm{i}}$ & $\begin{array}{c}F_{\text {int }} \\
\left(\mathrm{mK} \mathrm{km} \mathrm{s}^{-1}\right)\end{array}$ & $\begin{array}{c}N_{\text {tot }} \\
\left(\mathrm{cm}^{-2}\right)\end{array}$ \\
\hline${ }^{13} \mathrm{CO}(5-4)$ & 550926.30 & 79 & 5.0 & $480(50)$ & 5 & $+1.8(0.1)$ & $3.4(0.1)$ & - & $2023(31)$ & $0.4-1.010^{16}$ \\
\hline $\mathrm{H}^{37} \mathrm{Cl}(1-0) \quad F=3 / 2-3 / 2$ & 624964.37 & 30 & 1.3 & $\leq 15$ & 5 & - & - & - & - & - \\
\hline $\mathrm{H}^{37} \mathrm{Cl}(1-0) \quad F=5 / 2-3 / 2$ & 624977.82 & 30 & 2.0 & $\leq 15$ & 5 & - & - & - & - & - \\
\hline $\mathrm{H}^{37} \mathrm{Cl}(1-0) \quad F=1 / 2-3 / 2$ & 624988.33 & 30 & 0.7 & $\leq 15$ & 5 & - & - & - & - & - \\
\hline $\mathrm{H}^{35} \mathrm{Cl}(1-0) \quad F=3 / 2-3 / 2$ & 625901.60 & 30 & 1.3 & $27(6)$ & 6 & - & - & - & $70(14)$ & - \\
\hline $\mathrm{H}^{35} \mathrm{Cl}(1-0) \quad F=5 / 2-3 / 2$ & 625918.76 & 30 & 2.0 & $35(6)$ & 6 & $+1.7(0.2)$ & $2.8(0.3)$ & $0.1(0.9)$ & $114(14)$ & $210^{13}$ \\
\hline $\mathrm{H}^{35} \mathrm{Cl}(1-0) \quad F=1 / 2-3 / 2$ & 625932.01 & 30 & 0.7 & $\leq 18$ & 6 & - & - & - & - & $\begin{array}{l}1 \\
-\quad 0\end{array}$ \\
\hline
\end{tabular}

${ }^{a}$ Frequencies and spectroscopic parameters have been extracted from the Jet Propulsion Laboratory molecular database (Pickett et al. 1998).

${ }^{\mathrm{b}}$ At a spectral resolution of $1 \mathrm{~km} \mathrm{~s}^{-1}$. 


\section{4. $\mathrm{HCl}$ excitation and abundance calculations}

To fit the $\mathrm{H}^{35} \mathrm{Cl}$ (hereafter $\mathrm{HCl}$ ) $J=1-0$ spectrum, we used the GILDAS CLASS tool to fit the $\mathrm{HCl}(1-0)$ spectrum, which gives the best fit of the hyperfine components providing four parameters: (i) the LSR velocity, (ii) the linewidth (FWHM), (iii) the sum of the opacity at the central velocities of all the hyperfine components $p_{1}=\sum_{\mathrm{i}} \tau_{\mathrm{i}}$, and (iv) the

product $p_{2}=p_{1} \times\left[J\left(T_{\mathrm{ex}}\right)-J\left(T_{\mathrm{bg}}\right)-J\left(T_{\mathrm{c}}\right)\right]$, where $J(T)=\frac{h \nu / k}{\mathrm{e}^{h \nu / k T}-1}$ and $T_{\mathrm{c}}$ is the temperature of the continuum emission. In the L1157-B1 case, $J\left(T_{\mathrm{c}}\right.$ (no dust-continuum so far detected towards B1, e.g. Codella et al. 2009) can be neglected. Hence:

$$
T_{e x}=\frac{h \nu}{k}\left[\ln \left(1+\frac{h \nu}{k} \frac{p_{1}}{p_{2}}\right)\right]^{-1} .
$$

The red curve in Fig. 1 shows the fit obtained with the CLASS tool, which gives the fit parameters reported in Table 1 . In the optical thin case, the relative intensity ratio between the three hyperfine lines is 1:3:2 and it significatively diverges for opacities larger than $\sim 1$. The undetected weakest hyperfine component $F=1 / 2-1 / 2$ indicates that the opacity of the main hyperfine component $(F=5 / 2-3 / 2)$ is lower than 1 . Since the derived $T_{\text {ex }}$ depends on the opacity and the assumed source extent (to reproduce the observed $T_{\mathrm{mb}}$ ), we derived three possible solutions assuming different source sizes. In fact, L1157-B1 is a well-known clumpy arch-like structure, with a whole size around $15^{\prime \prime}$ (e.g. the $\mathrm{CH}_{3} \mathrm{CN}$ map reported by Codella et al. 2009). The emission at the highest velocities (more than $20 \mathrm{~km} \mathrm{~s}^{-1}$ with respect to the ambient velocity), comes from a smaller (less than 10") region of L1157-B1 (Gueth et al. 1998). The $\mathrm{HCl}$ line profile, although observed with a low $\mathrm{S} / \mathrm{N}$, seems to exclude the association with high-velocity wings. Thus, we assumed three cases: 10", 15", and extended emission (i.e. a filling factor equal to unity). The latter case allows us to verify whether the $\mathrm{HCl}$ emission comes from the parent molecular cloud. Assuming the highest possible line opacity $(\tau=1)$, the derived minimum $T_{\text {ex }}$ values are: $5 \mathrm{~K}$ (extended), and $\sim 7 \mathrm{~K}\left(10^{\prime \prime}-15^{\prime \prime}\right)$. As previously reported, lower line opacities are in principle also possible. Assuming $\tau=0.01$ excitation temperatures of $18 \mathrm{~K}$ (extended) or $\sim 68 \mathrm{~K}\left(10^{\prime \prime}-15^{\prime \prime}\right)$ are obtained.

The $\mathrm{HCl}(1-0)$ transition is associated with a critical density larger than $4 \times 10^{7} \mathrm{~cm}^{-3}$ for temperatures larger than $10 \mathrm{~K}$ (Neufeld \& Green 1994) and the upper level energy is $\sim 30 \mathrm{~K}$. Thus, the corresponding line emission is expected to trace dense and relatively warm gas. Figure 3 shows $T_{\text {kin }}$ versus density for different columun density $(\mathrm{N}(\mathrm{HCl}))$ values, obtained with a non-LTE LVG code (Ceccarelli et al. 2003) using the Neufeld \& Green (1994) collisional coefficients and the Einstein coefficients quoted in the Jet Propulsion Laboratory molecular database (Pickett et al. 1998). The Neufeld \& Green collisional coefficients for $\mathrm{HCl}-\mathrm{He}$ are usually scaled by a factor 1.38 to take into account the reduced mass ratio with 
$\mathrm{H}_{2}$. Larger factors might actually apply, as observed for other light hydrides (e.g. HF- $\mathrm{H}_{2}$, Guillon et al. 2008). The collisional coefficients can be significantly different for $\mathrm{H}_{2}$ in $J=0$ and in $J>0$. As it is difficult to quantify the differences between $\mathrm{HCl}-\mathrm{He}$ and $\mathrm{HCl}-\mathrm{H}_{2}$, and to avoid the introduction of additional free parameters (e.g. the $\mathrm{H}_{2}$ ortho-to-para ratio), we have simply scaled the $\mathrm{HCl}-\mathrm{He}$ rates by the standard factor of 1.38. This implies that the derived $\mathrm{N}(\mathrm{HCl})$ may be an overestimate, a result that does not affect the major conclusion of this study. The opacity is assumed to range from 0.01 to 1 , accordingly to the fit of the $\mathrm{HCl}(1-0)$ spectrum. Dashed and solid contours indicate the solutions for the two extreme sizes, extended and 10", respectively. We used the modelling approach presented in Daniel, Cernicharo \& Dubernet (2006), who showed that, for the $\mathrm{HCl}(1-0)$ line (see their Fig. 6), the differences between the opacities as determined with and without taking into account collisional and radiative transitions between hyperfine levels are negligibles. The hyperfine treatment is thus not necessary. From Fig. 3, the $\mathrm{HCl}(1-0)$ emission cannot arise from the molecular cloud, as the extended emission and cold hypothesis would require a cloud density of at least $4 \times 10^{6} \mathrm{~cm}^{-3}$. On the contrary, a source size of $10^{\prime \prime}$ would require densities larger than $2 \times 10^{5} \mathrm{~cm}^{-3}$ and temperatures larger than $\sim 15 \mathrm{~K}$ (the larger the temperature the smaller the gas density). The $\mathrm{HCl}$ column density has to be at least a few $10^{12} \mathrm{~cm}^{-2}$, while its maximum value, corresponding to an opacity of 1 , is $2 \times 10^{13} \mathrm{~cm}^{-2}$. In order to further constrain the LVG solutions and to estimate the $\mathrm{HCl}$ abundance, we evaluated the $\mathrm{H}_{2}$ column density using the ${ }^{12} \mathrm{CO}(5-4)$, from Lefloch et al. (2010), and ${ }^{13} \mathrm{CO}(5-4)$ lines (present paper). From the ratio of the main-beam brightness temperatures, the derived opacities are 2.7 and 0.036 respectively (assuming $\left[{ }^{12} \mathrm{CO}\right] /\left[{ }^{13} \mathrm{CO}\right]=77$; Wilson \& Rood 1994). A non-LTE LVG code, with the Flower et al. (2001) collisional coefficients, was used to constrain the density, temperature and $\mathrm{CO}$ column density. For a source size of $10^{\prime \prime}$, we found $\mathrm{N}\left({ }^{13} \mathrm{CO}\right)=1$ $\times 10^{16} \mathrm{~cm}^{-2}, T_{\text {kin }}=200-250 \mathrm{~K}$ and $n_{\mathrm{H} 2}=0.2-1.0 \times 10^{6} \mathrm{~cm}^{-3}$. If we assume $15^{\prime \prime}$, we have $\mathrm{N}\left({ }^{13} \mathrm{CO}\right)=4 \times 10^{15} \mathrm{~cm}^{-2}, T_{\text {kin }}=120-150 \mathrm{~K}$ and $n_{\mathrm{H} 2}=1-5 \times 10^{5} \mathrm{~cm}^{-3}$. These measurements are consistent with previous estimates (Lefloch et al. 2010), and correspond to $\mathrm{N}\left(\mathrm{H}_{2}\right)=3-8$ $\times 10^{21} \mathrm{~cm}^{-2}$ assuming $\left[{ }^{12} \mathrm{CO}\right] /\left[\mathrm{H}_{2}\right]=1 \times 10^{-4}$ (Wilson \& Rood (1994). By using the the output of the $\mathrm{CO}(5-4)$ analysis in the $10^{\prime \prime}$ case, highlighted in the striped box of Fig. 3 , the $\mathrm{HCl}$ column density is likely to be $2 \times 10^{13} \mathrm{~cm}^{-2}$. Note that the same $\mathrm{N}(\mathrm{HCl})$ estimate is obtained by using a source size of $15^{\prime \prime}$.

In conclusion, assuming a source size of $10^{\prime \prime}-15^{\prime \prime}$, we obtain an abundance of $X(\mathrm{HCl})$ $\simeq 3-6 \times 10^{-9}$. This value is consistent with what found in high-mass star forming regions such as OMC-1 (from fews $10^{-10}$, Schilke et al. 1995 and Salez et al. 1996, to $2 \times 10^{-9}$, from Blake et al. 1985 and Neufeld \& Green 1994), W3A $\left(X\left(\mathrm{H}^{35} \mathrm{Cl}\right)=8 \times 10^{-10}\right.$; Cernicharo et al. 2010a) and a sample of 27 low- and high- mass protostars, where a $\mathrm{HCl}$ abundance in the $3-30 \times 10^{-10}$ range has been measured (Peng et al. 2010). 


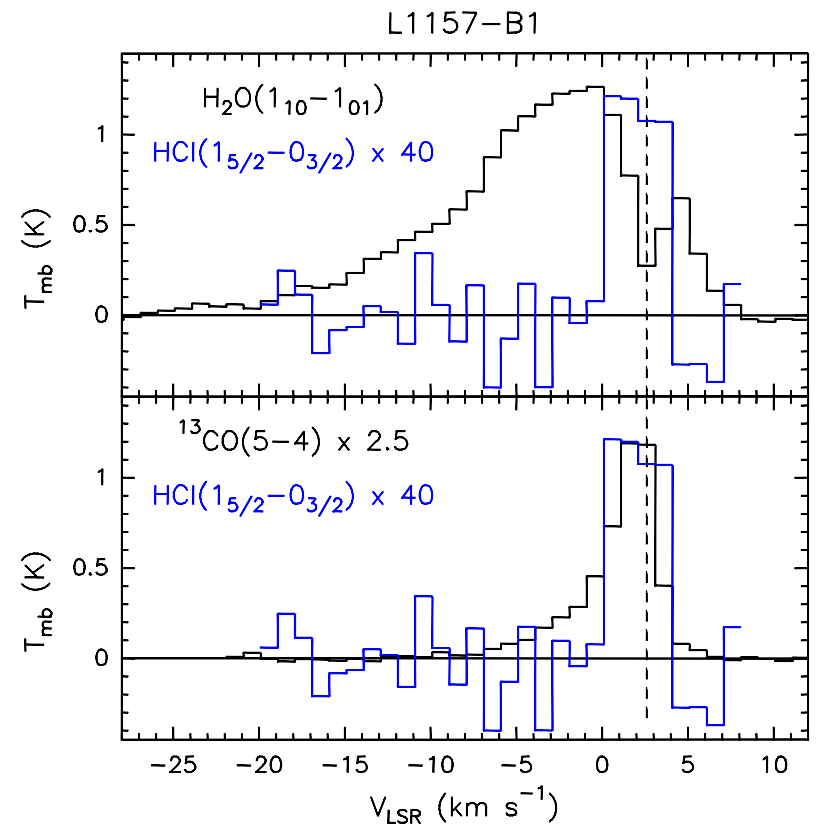

Fig. 2.- Comparison between the profiles of $\mathrm{H}^{35} \mathrm{Cl}(1-0) \quad F=5 / 2-3 / 2$, with those due to $\mathrm{H}_{2} \mathrm{O}\left(1_{10}-1_{01}\right)$, from Lefloch et al. (2010) and Codella (2010), and ${ }^{13} \mathrm{CO}(5-4)$. The $\mathrm{H}^{35} \mathrm{Cl}$ and ${ }^{13} \mathrm{CO}$ profile have been scaled for a direct comparison with the brighter $\mathrm{H}_{2} \mathrm{O}$ emission. The vertical solid line indicates the ambient LSR velocity $\left(+2.6 \mathrm{~km} \mathrm{~s}^{-1}\right.$ from $\mathrm{C}^{18} \mathrm{O}$ emission, Bachiller \& Peréz Gutiérrez 1997). 


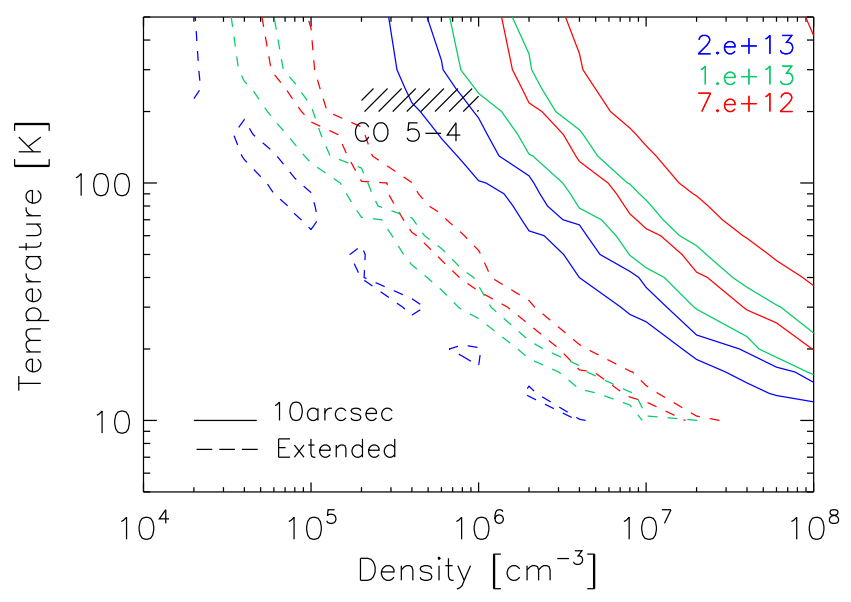

Fig. 3. - Kinetic temperature $T_{\text {kin }}$ of the $\mathrm{HCl}(1-0)$ transition versus gas density for different $\mathrm{HCl}$ column densities, as marked in the plot with different colours. The derived values of $\mathrm{N}(\mathrm{HCl})$ assuming different source sizes are showed in solid $\left(10^{\prime \prime}\right)$ and dashed lines (extended emission). Contours represent $\chi^{2}=2.7$ which corresponds to a goodness of $90 \%$ between the observed and modeled $\mathrm{HCl}(1-0)$ line temperatures. According to the fit of the $\mathrm{HCl}(1-0)$ spectrum the opacities range from 1 to 0.01 . The striped box individuates the solutions provided by the analysis of the ambient $\mathrm{CO}(5-4)$ emission (see text). 


\section{Chlorine chemistry in L1157-B1}

The shock chemistry in L1157-B1 has been very recently investigated by Viti et al. (2011) by the use of the chemical model UCL_CHEM coupled with a parametric shock model (Jimenez-Serra et al. 2008). We use their model to investigate the origin of $\mathrm{HCl}$. UCL_CHEM is a gas-grain chemical model with a two phases calculation. During Phase I, gravitational collapse (from a diffuse and atomic gas, with the final density being a free parameter), gas-phase chemistry and sticking onto dust particles with subsequent processing (mainly hydrogenation) occur. This phase simulates the formation of high density clumps or cores and starts from a fairly diffuse $\left(\sim 100 \mathrm{~cm}^{-3}\right)$ medium in atomic form (apart from a fraction of hydrogen in $\mathrm{H}_{2}$ ). Phase II is used to compute the time dependent chemical evolution of the gas and dust once the clump has formed and stellar activity is present (in the form of a protostar and/or outflows). In Viti et al. (2011) the UCL_CHEM was coupled with the parametric C-type shock model developed by Jimenez-Serra et al. (2008). Full details of the code can be found in Viti et al. (2004, 2011).

We used the same grid of models as in Viti et al. (2011) but we include chlorine gas and surface chemistry and we vary the initial elemental abundance of $\mathrm{Cl}$ (from solar to depleted by a factor of 200 - e.g. Schilke et al. 1985). In order to reproduce the observed $\mathrm{HCl}$ abundance $\left(\sim 10^{-9}\right)$ : (i) the initial elemental abundance of $\mathrm{Cl}$ needs to be depleted by a factor of $\sim 200$ with respect to its solar value (see also Cernicharo et al. 2010a); (ii) $\mathrm{HCl}$ is formed in the gas phase (or at least surface reactions do not seem to be needed to produce it); (iii) the $\mathrm{HCl}$ abundance is independent on the shock parameters and does not form nor is destroyed during the shock phase. This is consistent with the observed $\mathrm{HCl}$ line profiles, with no clear hints of high-velocity wings (Fig. 2).

\section{Conclusions}

We presented the first detection of hydrogen chloride in a protostellar shock. The $\mathrm{HCl}$ line originates in the $10^{\prime \prime}-15^{\prime \prime}$ region observed by the interferometric observations at PdBI (Codella et al. 2009), from a warm $(>120 \mathrm{~K})$ and dense $\left(\sim 10^{5}-10^{6} \mathrm{~cm}^{-3}\right)$ gas. Using the $\mathrm{H}_{2}$ column density derived from the $\mathrm{CO}(5-4)$ line observations, we derive a $\mathrm{HCl}$ fractional abundance of $3-6 \times 10^{-9}$. This is consistent with that observed towards low- and high- mass protostars. Modelling of C-type shocks suggests no increase of $\mathrm{HCl}$ associated with the shock, in agreement with the $\mathrm{HCl}$ line profile not showing high velocities $(\geq 20$ $\mathrm{km} / \mathrm{s}$ ) wings. In conclusion, $\mathrm{HCl}$ emission has been detected because of the increase of the corresponding column density as a result of shock compression, but it is independent on the passage of the shock. This result shows that in the bow shock L1157-B1, used as a laboratory 
where to investigate the effects of shocks driven by low-mass protostars, either $\mathrm{HCl}$ is not present in the volatile grain mantles or, as suggested by Schilke et al. (1995) for the highmass star forming region OMC-1, hydrogen chloride remains locked on the refractory dust even after the passage of a shock. Both hypothesis seem, however, implausible as previous observations have clearly demonstrated that the shock in L1157-B1 is strong enough (i) to release molecular species from the mantles (e.g. $\mathrm{H}_{2} \mathrm{O}, \mathrm{NH}_{3}$; Lefloch et al. 2010, Codella et al. 2010), as well as (ii) to destroy the core of the dust grains, as testified by the large increase of $\mathrm{SiO}$ emission (e.g. Bachiller et al. 2001). The lack of enhanced $\mathrm{HCl}$ therefore rather suggests that chlorine is elsewhere than in $\mathrm{HCl}$, against all previous chemical models, or (and this seems even less plausible) that chlorine is less abundant in the L1157 region than what is usually assumed. Evidently, the present observations represent a puzzle. Similar observations in other shocked regions will be necessary to solve it out.

HIFI has been designed and built by a consortium of institutes and university departments from across Europe, Canada and the United States under the leadership of SRON Netherlands Institute for Space Research, Groningen, The Netherlands and with major contributions from Germany, France and the US. Consortium members are: Canada: CSA, U.Waterloo; France: CESR, LAB, LERMA, IRAM; Germany: KOSMA, MPIfR, MPS; Ireland, NUI Maynooth; Italy: ASI, IFSI-INAF, Osservatorio Astrofisico di Arcetri-INAF; Netherlands: SRON, TUD; Poland: CAMK, CBK; Spain: Observatorio Astronómico Nacional (IGN), Centro de Astrobiología (CSIC-INTA). Sweden: Chalmers University of Technology - MC2, RSS \& GARD; Onsala Space Observatory; Swedish National Space Board, Stockholm University - Stockholm Observatory; Switzerland: ETH Zurich, FHNW; USA: Caltech, JPL, NHSC. We thank J. Cernicharo and V. Wakelam for instructive suggestions. This work has been supported by l'Agence Nationale pour la Recherche (ANR), France (project FORCOMS, contracts ANR-08-BLAN-022) and the Centre National d'Etudes Spatiales (CNES). We also thank many funding agencies for financial support. C.Codella and C.Ceccarelli acknowledge the financial support from the COST Action CM0805 "The Chemical Cosmos" and the French spatial agency CNES.

\section{References}

Anders E., \& Grevesse N. 1989, GeCoA 53, 197

Bachiller R., \& Peréz Gutiérrez M. 1999, ApJ 487, L93

Bachiller R., Peréz Gutiérrez M., Kumar M.S.N., \& Tafalla M. 2001, A\&A 372, 899

Benedettini M., Viti S., Codella C., et al. 2007, MNRAS 381, 1127 
Blake G.A., Keene J., \& Phillips T.G. ApJ 295, 501

Ceccarelli C., Maret S., Tielens A.G.G.M., Castets A., \& Caux E. 2003, A\&A 410, 587

Ceccarelli C., Bacmann A., Boogert A., et al. 2010, A\&A 521, L22

Cernicharo J., Goicoechea J.R., Daniel F., et al. 2010a, A\&A 518, L115

Cernicharo J., Decin L., Barlow M.J., et al. 2010b, A\&A 518, L136

Codella C., Benedettini M., Beltrán M.T., et al. 2009, A\&A 507, L25

Codella C., Lefloch B., Ceccarelli C., et al. 2010, A\&A 518, L112

Daniel F., Cernicharo J., \& Dubernet M.-L. 2006, ApJ 648, 471

Flower D.R. 2001 JPhB 34, 2731

Garay G., Köhnenkamp I., Bourke T.L., Rodríguez L.F., \& Lehtinen K.K. 1998, ApJ 509, 768

de Graauw Th., Helmich F.P., Phillips T.G., et al. 2010, A\&A 518, L6

Gueth F., Guilloteau S., \& Bachiller R. 1996, A\&A 307, 891

Gueth F., Guilloteau S., \& Bachiller R. 1998, A\&A 333, 287

Guillon G., Stoecklin T., Voronin A., \& Halvick P. 2008, JChPh. 129, 4308

Jiménez-Serra I., Caselli P., Martín-Pintado J., \& Hartquist T.W. 2008, A\&A 482, 549

Lefloch B., Cabrit S., Codella C., et al. 2010, A\&A 518, L113

Liseau R., Ceccarelli C., Larssson B. et al. 1996, A\&A 315, L181

Lodders K., \& Palme H. 2009, M\&PSA 72, 5154

Neufeld D.A., \& Green S. 1994, ApJ 432, 158

Neufeld D.A., Nisini B., Giannini T., et al. 2009, ApJ 706, 170

Neufeld D.A., \& Wolfire M.G. 2009, ApJ 706, 1594

Peng R., Yoshida H., Chamberlin R.A., et al. 2010, ApJ 723, 218

Pickett H.M., Poynter R.L., Cohen E.A., Delitsky M.L., Pearson J.C., and Müller H.S.P. 1998, J. Quant. Spectrosc. \& Rad. Transfer 60, 883

Salez M., Frerking M.A., \& Langer D.A. 1996, ApJ 467, 708

Schilke P., Phillips T.G., \& Wang N. 1995, ApJ 441, 334

Schöier F.L., van der Tak FFS., van Dishoeck E.F., \& Black J.H. 2005, A\&A, 432, 369

Van der Tak F.F.S., Black J.H., Schöier F.L., Jansen D.J., \& van Dishoeck, E.F. 2007, A\&A 468,627

Viti S., Collings M.P., Dever J.W., McCoustra M.R.S., \& Williams D.A. 2004, MNRAS 354, 1141

Viti S., Jiménez-Serra I., Yates J.A., et al. 2011 ApJ 740, L3

Wilson T.L. \& Rood R. 1994, ARA\&A 32, 191 